Salma Mawfek Khaled, Peter Haddad, Majid Al-Abdulla, Tarek Bellaj, Yousri Marzouki, Youssef Hasan, Ibrahim Al-Kaabi

Catalina Petcu, Lina Bader, Iman Amro, Marwa Al-Assi, Elham Kadkhodaei, Aisha Al-Hamadi, Mohammed Al-Thani, Peter Woodruff

\section{Background}

As COVID-19 pandemic was declared in March 2020 by the World Health Organization,

31 million cases worldwide and 1 million deaths were reported six months later (Worldometer 2020).

COVID-19 associated with high rates of stress, anxiety and depressive symptoms in the general population (Rajkumar 2020).

Psychiatric consequences of COVID-19 likely reflect sociodemographic variables, infection rates, culture and access to both physical and mental health services.

\section{Aims}

1. Identify risk factors and resilience factors for psychiatric symptoms during the pandemic

2. Increase understanding of the impact of the pandemic on mental health to address current and future crisis

3. Inform the planning and delivery of individualized treatment

\section{Methods}

\section{Data collection}

- Longitudinal online survey (English and Arabic) available at www.q-lamp.com

- SMS-based recruitment and social media recruitment (e.g. Facebook, Instagram, and Twitter)

- Online consent (English and Arabic)

- Completion time for questionnaires: approx. 20 to 35 minutes

- Baseline questionnaire with follow up at 3, 6, 9 and 12 months

- Study completion date: July 2021

\section{Target population}

- Citizens and expatriates

- 18 years old and above

- Read Arabic or English

\section{Instruments}

- Sociodemographic questionnaire including personal and family experience of COVID-19 infection

- Standard instruments to assess psychiatric morbidity including depression, anxiety and PTSD

- Research team-designed instruments to assess social impact of pandemic

- Standard questionnaires to assess resilience, personality, loneliness, religious beliefs and social networks

\title{
Preliminary results
}

Table 1. Percentage of Participants Endorsing Increased Psychological Symptoms during Pandemic

\begin{tabular}{|l|c|}
\hline $\begin{array}{l}\text { How different are these feelings to how } \\
\text { you felt before the pandemic? }\end{array}$ & $\begin{array}{c}\text { Little/Much worse } \\
\%\end{array}$ \\
\hline Depression Symptoms & 52 \\
\hline Anxiety Symptoms & 55 \\
\hline Post-Traumatic Stress Rating & 52 \\
\hline Loneliness Scale & \\
\hline Perceived Stress Scale & 41 \\
\hline
\end{tabular}

Figure 1. Percentage of Endorsed Socioeconomic Health, and Social Changes during Pandemic

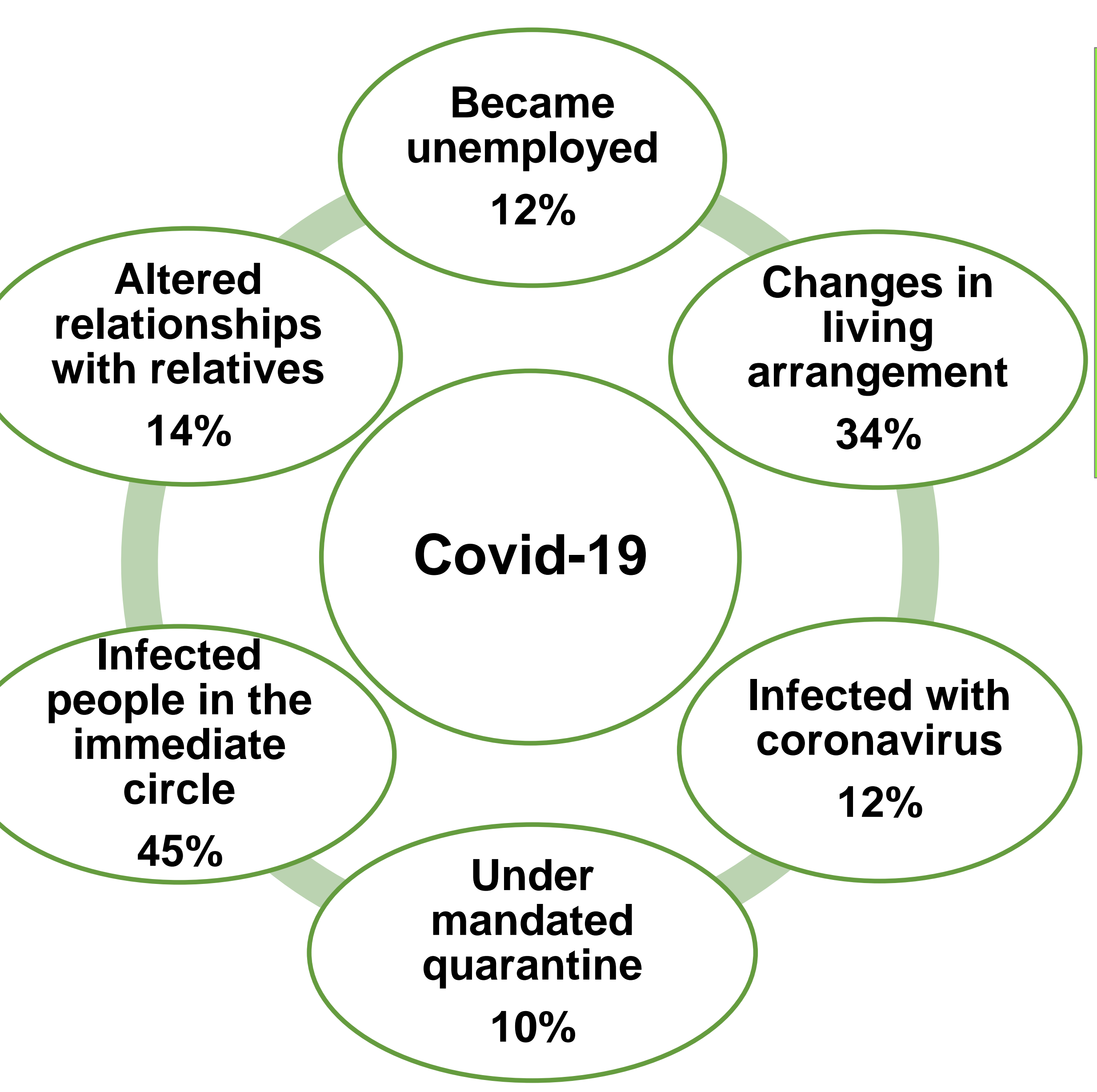

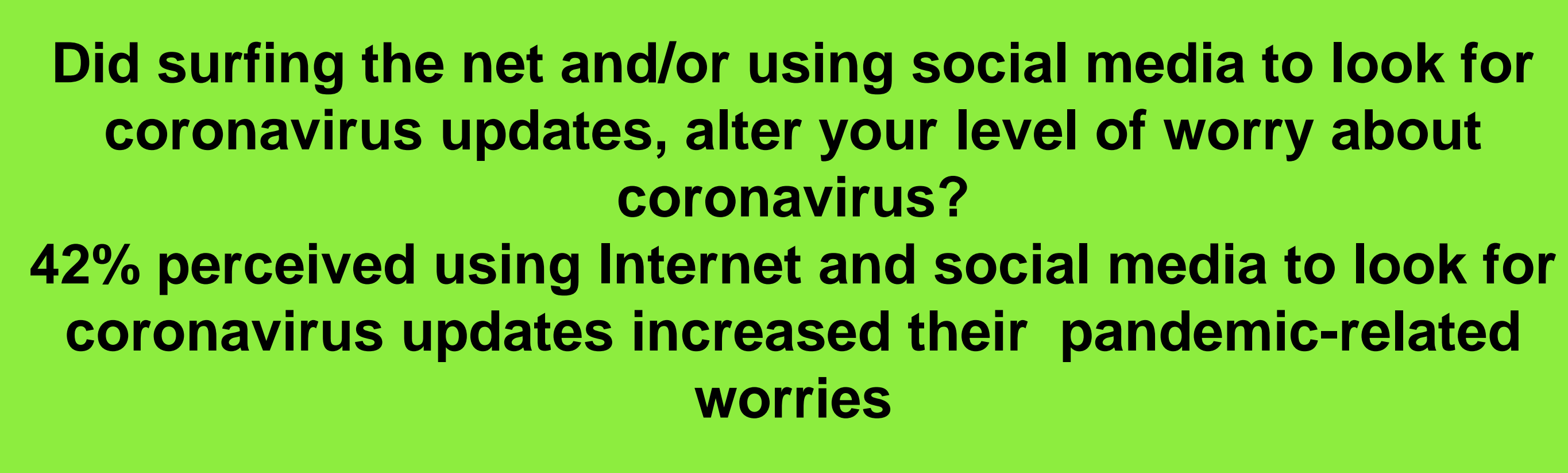

Of 181 completed surveys so far:

- $65 \%$ females and $35 \%$ males.

- $27 \%$ Qataris

- $52 \%$ married,

- $25 \%$ had Grade 12 or lower level of educational attainment

- $46 \%$ were unemployed.

\section{Future directions}

- COVID-19 affected personal health, living arrangements, employment, and health of family and friends.

- Approximately, $41 \%$ to $55 \%$ of respondents perceived post-COVID stress levels, mental health, and loneliness worsened than before the pandemic.

- The wide-availability of information about the pandemic on the internet and social media was perceived as source of pandemic-related worries

- The continued provision of mental health service and educational campaigns for effective stress and mental health management is warranted ir light of unremitting global pandemic crisis.

\section{Acknowledgements}

This study is supported by Qatar University Emergency Response Grant (QUERG-CAS-2020-1).

\section{References}

Rajkumar RP. COVID-19 and mental health: A review of the existing literature. Asian J Psychiatry. 2020;52:102066. doi:10.1016/j.ajp.2020.102066

Worldometers. Coronavirus. 2020. https://ipv6.worldometers.info/coronavirus/ 